\title{
Retrospective analysis of perioperative factors on outcome of patients undergoing surgery for Moyamoya disease
}

\author{
Navneh Samagh, Hemant Bhagat ${ }^{1}$, Vinod K. Grover ${ }^{1}$, Neeru Sahni ${ }^{1}$, Ashish Agarwal ${ }^{2}$, Sunil K. Gupta ${ }^{2}$ \\ Departments of Neuroanaesthesia, ${ }^{1}$ Anaesthesia and ${ }^{2}$ Neurosurgery, PGIMER, Chandigarh, India
}

\begin{abstract}
Background: The short term outcome of patients undergoing surgery for Moyamoya disease can be affected by various perioperative factors. However, due to lesser prevalence of this disease in our country, data relating the effect of perioperative factors on the overall neurological outcomes of these patients is lacking. Aims: To analyze the effect of perioperative factors on the duration of postoperative hospital stay in patients undergoing surgery for Moyamoya disease. Settings and Design: It is a retrospective study analyzing various perioperative factors influencing the overall outcome of patients undergoing surgery for Moyamoya disease at a tertiary care centre in North India. Methods and Material: The medical records of all patients who underwent revascularization surgeries for Moyamoya disease from 2007 to till January 2014 were included for retrospective analysis. Various preoperative, intraoperative, and postoperative data were recorded. The data was statistically compared for short and prolonged hospital stay for various perioperative factors. The duration of post operative hospital stay was categorized as short ( $<5$ days) and prolonged ( $>5$ days). Statistical Analysis: Kolmogrov Smirnov test was applied to see the normality of continuous data. The association of various categorically classified data with 2 groups was found using Fisher Exact test. The trends in intraoperative hemodynamics were analysed using 2 way repeated measure Anova test. $T$-test was used for comparing two group means for various parameters. Results: A total of 15 patients were included in the study. One patient underwent surgery twice on two different occasions. Thirteen patients belonged to paediatric age group ( $<18$ years). The type of anaesthetics used for induction and maintenance had no effect on patient outcome. Mean duration of anaesthesia was 2.45 (1.3-4.0) hours. The mean duration of hospital stay was 5.13 (3-10) days. Most of the parameters did not have significant effect on postoperative hospital stay. Patients with mean value of intraoperative end tidal carbon dioxide $\left(\mathrm{EtCO}_{2}\right)$ either less than $31 \mathrm{mmHg}$ or more than $35 \mathrm{mmHg}$ had statistically significant prolongation of hospital stay. Conclusion: Maintaining the intraoperative $\mathrm{EtCO}_{2}$ between $31-35 \mathrm{mmHg}$ may be associated with short hospital stay when compared to those who have intraoperative $\mathrm{EtCO}_{2}$ either less than $31 \mathrm{mmHg}$ or more than $35 \mathrm{mmHg}$.
\end{abstract}

Key words: Moyamoya disease, perioperative factors, postoperative hospital stay

\section{Introduction}

The word Moyamoya in Japanese means "something hazy, like a puff of cigarette smoke drifting in the air." This rare disease is characterized by worsening

\begin{tabular}{|l|l|}
\hline \multicolumn{2}{|c|}{ Access this article online } \\
\hline Quick Response Code: & Website: \\
\hline & www.ruralneuropractice.com \\
\cline { 2 - 2 } & \\
\hline & \\
\hline
\end{tabular}

stenosis of terminal portions of internal carotid artery, proximal portions of anterior and middle cerebral artery (MCA), sometimes involving the posterior cerebral arteries as well. Net like images on angiograms arise from the proliferated penetrating arteries at the base of the brain that are the main source of collateral flow to the ischemic areas of brain distal to the occlusion. ${ }^{[1]}$

We retrospectively analyzed the perioperative parameters of 15 patients of Moyamoya disease operated in our institute over last 7 years for their effect on the duration of postoperative hospital stay. The duration of postoperative hospital stay was categorized as

Address for correspondence:

Dr. Hemant Bhagat, Department of Anaesthesia, PGIMER, Chandigarh, India. E-mail: hembhagat@rediffmail.com 
short ( $<5$ days) and prolonged ( $>5$ days). The data were compared for the duration of hospital stay.

\section{Materials and Methods}

The medical records of all patients who underwent revascularization surgeries for Moyamoya disease from 2007 to January 2014 were analyzed retrospectively. Approval was sought from institutional ethics committee. The following preoperative, intraoperative, and postoperative data were recorded: Age, sex, weight, associated congenital anomalies, clinical features, radiological findings, medications, surgical procedure, first or repeat surgery, type of anesthesia, the induction agent used, the maintenance agent used, intraoperative hemodynamics, value of end-tidal carbon dioxide $\left(\mathrm{EtCO}_{2}\right)$, temperature, fluid requirement, blood loss, urine output, emergence, duration of surgery, duration of anesthesia, postoperative recovery, new onset neurological deficit, other complications such as pain, postoperative nausea and vomiting, respiratory complications, sepsis and duration of hospital stay. Primary outcome was the duration of hospital stay that was categorized as either short or prolonged hospital stay. Short stay was defined as $<5$ days and stay in hospital $>5$ days was taken as prolonged stay.

\section{Statistical analysis}

Kolmogrov-Smirnov test was applied to see the normality of continuous data. The association of various categorically classified data with 2 groups was found using Fisher Exact test. The trends in intraoperative hemodynamics were analyzed using 2 way repeated measure ANOVA test. $t$-test was used for comparing two group means for various parameters.

\section{Results}

Perioperative data were analyzed for 15 patients and 16 procedures. The mean age of the patients was $12.3(3-45)$ years with nine male and seven female patients. Mean weight was $28.7(11-70) \mathrm{kg}$. Baseline blood investigations were within normal range for all patients. Aspirin, antiepileptics and steroids were continued till the day of surgery. Three patients underwent myo-synangiosis (19\%), four underwent encephalo-duro-arterio-myo-synangiosis (EDAMS) $(25 \%)$, two had MCA bypass (12\%) and seven underwent a combination of MCA bypass and EDAMS (44\%) under general anesthesia.

Blood loss requiring transfusion was recorded in one patient and all patients had adequate intraoperative urine output. None of the patients had intraoperative hypothermia or hyperthermia. At the end of the procedure, all patients could be extubated, and the mean duration of anesthesia was 2.45 (1.3-4) h. One patient experienced postoperative nausea and vomiting but there was no delay in time to accept oral intake in any patient. Postoperatively, all patients were hemodynamically stable and none experienced severe pain. Among the neurological complications, two patients suffered from aphasia, one had associated new onset right upper limb monoparesis and one patient developed right fronto-temporo-parietal subdural effusion. Among other complications, urinary tract infection was reported in one patient.

Mean duration of hospital stay was 5.13 (3-10) days. Sex of the patient, congenital anomalies, type of induction agent used, type of anesthetic used for maintenance of anesthesia were not found to have any significant effect on the duration of hospital stay $(P>0.05)$. Clinical features including history of recurrent transient ischemic attack (TIA), seizures and motor weakness had a trend predicting prolonged duration of hospital stay though it was not statistically significant. Average $\mathrm{EtCO}_{2}$ value $<31 \mathrm{mmHg}$ throughout the surgery showed statistically significant prolongation of hospital stay when compared with patients with mean $\mathrm{EtCO}_{2}$ value between 31 and $35 \mathrm{mmHg}(P<0.0001)$. Furthermore, patients with mean $\mathrm{EtCO}_{2}$ value $>35 \mathrm{mmHg}$ had statistically significant prolongation of hospital stay when compared with those with $\mathrm{EtCO}_{2}$ value between 31 and $35 \mathrm{mmHg}$ throughout the procedure [Table 1]. The hemodynamic variables were maintained and similar in patients who had either short or prolonged hospital stay [Figures 1 and 2].

\section{Discussion}

The outcome of patients undergoing neurosurgery for Moyamoya disease depends on various perioperative factors. Patients presenting with a history of recurrent TIA, seizures and motor deficits are at high risk for postoperative ischemic complications. ${ }^{[2]}$ Furthermore, intraoperative maintenance of cerebral perfusion pressure is crucial for good postoperative neurological recovery. ${ }^{[3]}$ This is influenced by the type of anesthetic used, hemodynamics, fluid resuscitation, blood loss and hematocrit, $\mathrm{EtCO}_{2^{\prime}}$ temperature regulation, urine output and the type of surgical procedure being done. ${ }^{[4]}$

In our study, we did not find the effect of preoperative recurrent TIA, motor deficits and seizures on the duration of hospital stay. As the underlying 
pathophysiology predisposes these patients to ischemic complications, appropriate anesthetic techniques to maintain cerebral blood flow and cerebral perfusion pressure should be followed. There is no consensus or guideline for any specific agent to be used for anesthetic induction or maintenance in these cases as maintenance of cerebral blood flow and cerebral perfusion pressure is the ultimate goal,

Table 1: Effect of perioperative factors on duration of postoperative hospital stay

\begin{tabular}{|c|c|c|c|}
\hline Variable & $\begin{array}{l}\text { Short stay } \\
(<5 \text { days })\end{array}$ & $\begin{array}{c}\text { Prolonged stay } \\
\text { ( } \geq 5 \text { days })\end{array}$ & $P$ \\
\hline \multicolumn{4}{|l|}{$\overline{\text { Sex }}$} \\
\hline Male & 3 & 6 & 1.000 \\
\hline Female & 2 & 5 & \\
\hline \multicolumn{4}{|l|}{ Congenital anomalies } \\
\hline Present & 1 & 0 & 0.676 \\
\hline Absent & 4 & 11 & \\
\hline \multicolumn{4}{|l|}{ Clinical features } \\
\hline History of TIA & 0 & 3 & 0.791 \\
\hline Motor weakness & 3 & 7 & \\
\hline History of seizures & 1 & 5 & \\
\hline \multicolumn{4}{|l|}{ Induction agent } \\
\hline Thiopentone & 1 & 6 & 0.130 \\
\hline Propofol & 4 & 2 & \\
\hline Inhalational & 0 & 2 & \\
\hline \multicolumn{4}{|l|}{ Maintenance agent } \\
\hline Isoflurane & 2 & 7 & 0.228 \\
\hline Propofol & 0 & 2 & \\
\hline Desflurane & 3 & 1 & \\
\hline Sevoflurane & 0 & 1 & \\
\hline \multicolumn{4}{|l|}{ Intraoperative $\mathrm{EtCO}_{2}$} \\
\hline 31-35 & 4 & 1 & \\
\hline$<31$ & 0 & 2 & $<0.0001^{*}$ \\
\hline$>35$ & 1 & 8 & $<0.023^{\star *}$ \\
\hline
\end{tabular}

${ }^{*} P$ value between $\mathrm{EtCO}_{2}<31 \mathrm{mmHg}$ and normocapnia (31-35), ${ }^{* *} P$ value between $\mathrm{EtCO}_{2}>35 \mathrm{mmHg}$ and normocapnia (31-35). TIA: Transient ischemic attack, $\mathrm{EtCO}_{2}$ : End-tidal carbon dioxide

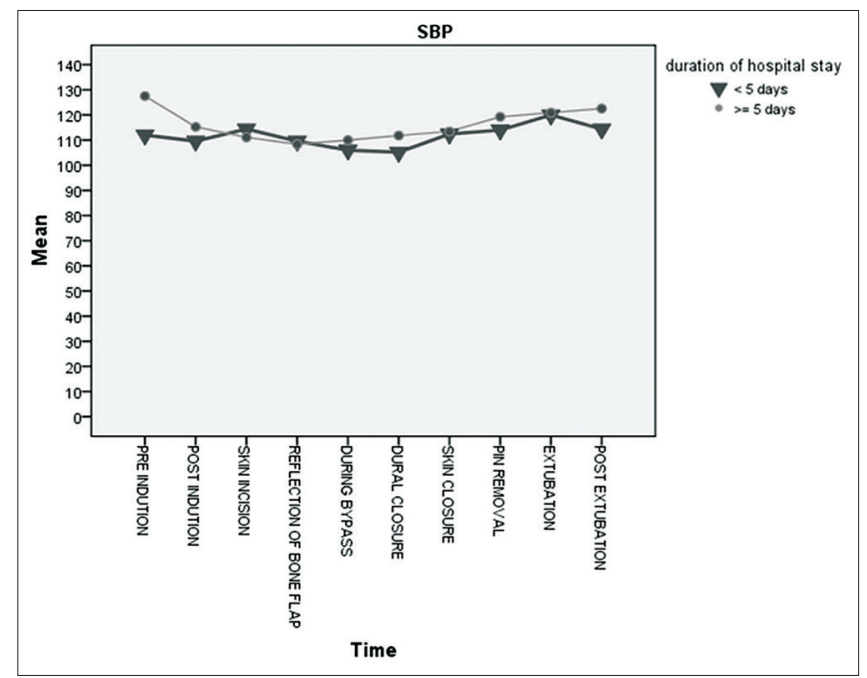

Figure 1: Systolic blood pressure at different time points of anaesthesia more so in pediatric patients. ${ }^{[5,6]}$ In our study, patients induced with propofol had a trend towards short hospital stay (4 days) whereas more proportion of patients who received thiopentone had a prolonged hospital stay ( $>5$ days), though this difference was not statistically significant.

The maintenance of normocarbia is essential to prevent postoperative ischemic complications as both hypocapnia and hypercapnia are detrimental in the presence of already diseased vessels. ${ }^{[7]}$ In our study, patients with normocapnia had shorter hospital stay when compared to those with hypocapnia or hypercapnia intraoperatively and the difference was found to be statistically significant. In a study by Chiu et al., decreased regional cerebral blood flow was observed in all patients when the $\mathrm{EtCO}_{2}$ decreased $<29 \mathrm{mmHg} .{ }^{[8]}$

We observed that the choice of anesthetic agent used to maintain depth of anesthesia did not have any effect on the duration of hospital stay. Summors et al. reported better preservation of autoregulation during sevoflurane than isoflurane anesthesia. ${ }^{[9]}$ In another study, Sato et al . concluded that regional cortical blood flow ( $\mathrm{rCoBF}$ ) may be decreased with inhalation anesthesia which may in turn provoke intracerebral steal phenomenon when compared to total intravenous anesthesia (TIVA). ${ }^{[10]}$ In contrast, certain studies have not shown any significant difference between TIVA and use of isoflurane. Kikuta et al. reported high $\mathrm{rCoBF}$ and low intracranial pressure with the use of propofol when compared with sevoflurane. ${ }^{[1]]}$ Perhaps maintaining adequate depth of anesthesia, especially during airway manipulation and at the time of revascularizaton may be more important than the type of anesthetic agent used.

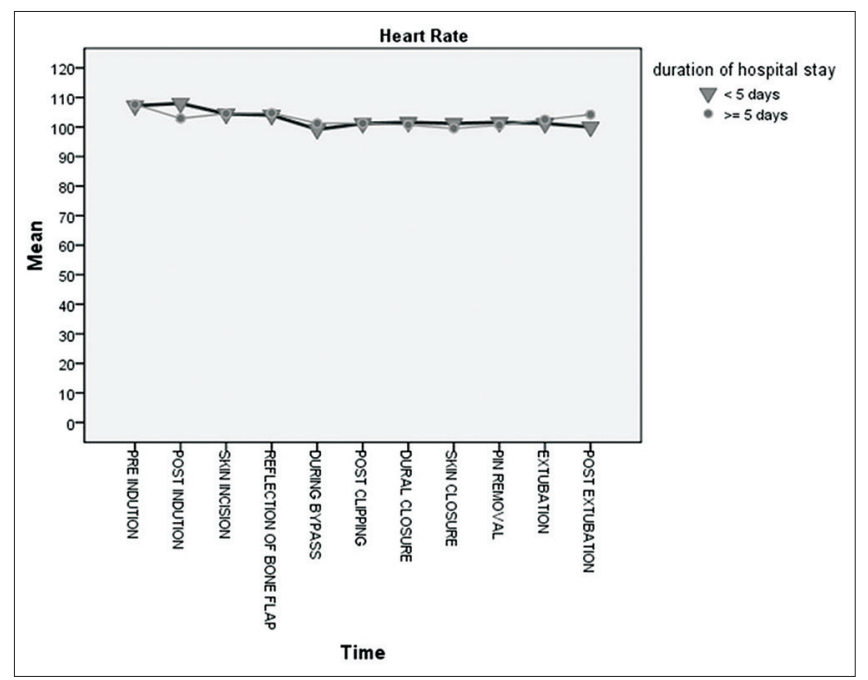

Figure 2: Heart rate at different time points of anaesthesia 
Adequate hydration is the single most important intervention preventing decrease in cerebral blood flow, and it was adequately done for all our patients. ${ }^{[5]}$ We analyzed that despite maintenance of mean arterial blood pressure, adequate depth of anesthesia and adequate fluid resuscitation in our patients, two patients suffered from aphasia, one had associated new onset right upper limb monoparesis and one patient developed right fronto-temporo-parietal subdural effusion. The patient who developed subdural effusion received thiopentone for induction, had lowest $\mathrm{EtCO}_{2}$ value recorded intraoperatively $(27 \mathrm{mmHg})$ and also, had highest duration of hospital stay (10 days). One patient who developed aphasia had undergone myosynangiosis. This patient had preoperative motor deficit, received thiopentone for induction with $\mathrm{EtCO}_{2}$ levels were between 34 and $37 \mathrm{mmHg}$ intraoperatively.

The present study concludes that maintaining the intraoperative $\mathrm{EtCO}_{2}$ between 31 and $35 \mathrm{mmHg}$ may be associated with early hospital discharge when compared to patients who have mean intraoperative $\mathrm{EtCO}_{2}$ either $<31$ or $>35 \mathrm{mmHg}$.

As this disease is not common in our country, only few patients underwent surgery at our institute in last 7 years. Hence, the data could be obtained on a small patient population. A prospective study in the future with larger sample size may yield more information on this rare but important entity.

\section{References}

1. Suzuki J, Takaku A. Cerebrovascular "Moyamoya" disease. Disease showing abnormal net-like vessels in base of brain. Arch Neurol 1969;20:288-99.

2. Sakamoto T, Kawaguchi M, Kurehara K, Kitaguchi K, Furuya H, Karasawa J. Risk factors for neurologic deterioration after revascularization surgery in patients with Moyamoya disease. Anesth Analg 1997;85:1060-5.

3. Soriano SG, Sethna NF, Scott RM. Anesthetic management of children with Moyamoya syndrome. Anesth Analg 1993;77:1066-70.

4. Parray T, Martin TW, Siddiqui S. Moyamoya disease: A review of the disease and anesthetic management. J Neurosurg Anesthesiol 2011;23:100-9.

5. Smith ER, Scott RM. Surgical management of Moyamoya syndrome. Skull Base 2005;15:15-26.

6. Kuwabara Y, Ichiya Y, Otsuka M, Tahara T, Gunasekera R, Hasuo K, et al. Cerebral hemodynamic change in the child and the adult with Moyamoya disease. Stroke 1990;21:272-7.

7. Iwama T, Hashimoto N, Yonekawa Y. The relevance of hemodynamic factors to perioperative ischemic complications in childhood Moyamoya disease. Neurosurgery 1996;38:1120-5.

8. Chiu D, Shedden P, Bratina P, Grotta JC. Clinical features of Moyamoya disease in the United States. Stroke 1998;29:1347-51.

9. Summors AC, Gupta AK, Matta BF. Dynamic cerebral autoregulation during sevoflurane anesthesia: A comparison with isoflurane. Anesth Analg 1999;88:341-5.

10. Sato K, Shirane R, Kato M, Yoshimoto T. Effect of inhalational anesthesia on cerebral circulation in Moyamoya disease. J Neurosurg Anesthesiol 1999;11:25-30.

11. Kikuta K, Takagi Y, Nozaki K, Yamada K, Miyamoto S, Kataoka H, et al. Effects of intravenous anesthesia with propofol on regional cortical blood flow and intracranial pressure in surgery for Moyamoya disease. Surg Neurol 2007;68:421-4.

How to cite this article: Samagh N, Bhagat H, Grover VK, Sahni N, Agarwal A, Gupta SK. Retrospective analysis of perioperative factors on outcome of patients undergoing surgery for Moyamoya disease. J Neurosci Rural Pract 2015;6:262-5.

Source of Support: Nil. Conflict of Interest: None declared. 